\title{
Ver, oír y aprender. Una filmación educativa para la enseñanza de habilidades en exploración física
}

\author{
Jordi Delás, Wilma Penzo, Antoni Delás, Raquel González-Cardona, César Morcillo, Gemma Martín
}

Introducción. Hemos desarrollado un cortometraje educativo para la formación en exploración física de estudiantes de medicina de tercer año, sin experiencia previa en habilidades clínicas.

Materiales y métodos. El estudio se ha realizado en el Servicio de Medicina Interna del Hospital Universitari Sagrat Cor de Barcelona durante dos años consecutivos. Se efectuaron evaluaciones sobre exploración física de los estudiantes antes y después de ver la película. La primera evaluación se llevó a cabo el primer día de estancia de los estudiantes en el hospital. A continuación, visualizaron la filmación y se les informó de que al cabo de 48 horas se efectuaría una segunda evaluación en la que deberían realizar una exploración física de acuerdo con lo que habían visto y oído en el cortometraje. Al final del período de seis semanas de estancia en el hospital se realizó una tercera evaluación. Todas las evaluaciones se llevaron a cabo por el mismo profesor, a partir de un listado de contenidos evaluativos elaborado previamente.

Resultados. Después de ver la película, 48 horas después de su llegada al hospital, los estudiantes habían mejorado en las diferentes pruebas de exploración física, en una tasa media del $43,4 \%$. Al cabo de seis semanas, se apreció un $14,3 \%$ de mejora en relación con la segunda evaluación del tercer día del curso.

Conclusión. Un cortometraje es un buen medio para la formación en la exploración física normal, más rápido que otros sistemas de enseñanza y favorece la adopción de competencias estables.

Palabras clave. Competencias clínicas. Educación médica. Evaluación de la educación. Exploración física. Paciente simulado. Películas y videos educativos.

Watch, listen and learn. An educational film for teaching physical examination skills

Introduction. We created a short educational film to teach third-year medical students on physical examination, without previous experience in clinical skills. Designed to be understood without other explanations than those which appear in the film, the students are shown the film on their first day in the hospital.

Materials and methods. The study has been made in the Department of Internal Medicine at the University Hospital Sagrat Cor of Barcelona during two consecutives course years. We assess the educational possibilities of this resource testing student skills before and after watching the film. The first evaluation was held on the students' first day in the hospital and they were informed that 48 hours later they would be given a second evaluation where they had to do a physical exploration according to the film. At the end of the 6-week period in the internal medicine department the third evaluation was given. All of these explorations were performed by the same professor with a same check list.

Results. After watching the film, 48 hours after their arrival, the students had improved in the different tests on physical exploration, at an average rate of $43.4 \%$. At the end of the stage their progression was of $14.3 \%$ improvement in relation to the third day of the course.

Conclusion. A short film is a good element for training in normal physical exploration, faster than other teaching systems, and also gives permanent skills.

Key words. Clinical competence. Educational measurement. Instructional films and videos. Medical education. Patient simulation. Physical examination.
Departamento de Medicina; Universitat de Barcelona; Hospital Universitari Sagrat Cor (J. Delás, R. González-Cardona). Departamento de Psiquiatría y Psicobiología Clínica; Universitat de Barcelona (W. Penzo, A. Delás). Departamento de Medicina; CIMA (C. Morcillo). Hospital del Mar (G. Martín). Barcelona, España.

Correspondencia: Dr. Jordi Delás. Hospital Universitari Sagrat Cor. Viladomat, 288 E-08029 Barcelona.

Fax: +34934948906

E-mail:

jdelas@ub.edu

Conflicto de intereses: No declarado.

Competing interests: None declared.

(C) 2014 FEM 


\section{Introducción}

La enseñanza de la medicina en las áreas clínicas es una formación impactante, rica en experiencias y emociones [1]. Sin embargo, son escasos los estudios dirigidos a enseñar habilidades clínicas de forma eficaz $[2,3]$.

En este sentido, hemos desarrollado un cortometraje educativo para la enseñanza de la exploración física, destinada a ser comprendida sin otras explicaciones que aquellas que aparecen en la filmación.

Los estudiantes visualizan la película, de menos de 10 minutos de duración, en su primer día en el hospital. En ella se recoge la continuidad de los procedimientos y la economía de movimientos del explorador y se constata que una exploración efectiva se puede hacer en un período.

Evaluamos las posibilidades educativas de este recurso mediante tests antes y después de ver la película.

\section{Materiales y métodos}

El estudio se ha realizado en el Servicio de Medicina Interna del Hospital Universitari Sagrat Cor, Universitat de Barcelona, durante dos cursos académicos: 2005-2006 y 2006-2007. Han participado profesores de medicina, profesionales de la comunicación audiovisual y un joven actor adiestrado a representar el papel de una persona que acude al médico para someterse a una revisión médica. Se han seguido una serie de etapas: elaboración de un listado de elementos formativos que se quieren transmitir en la exploración física y filmación de una exploración física en la práctica clínica, de alrededor de 10 minutos, donde se recogen estos elementos formativos, destinada a ser visualizada por los profesores y alumnos en el curso de un seminario. En ella se reproduce la exploración física general (abdomen, tórax anterior y posterior) desde el momento en el que se invita al paciente a pasar a la camilla hasta que se le despide. Se acompaña de elementos comunicativos, bajo el pretexto de explicar al paciente todo lo que se va a hacer.

La evaluación se ha realizado a los estudiantes en el primer día en el hospital. Se les pide que realicen una exploración física general de un compañero de grupo de acuerdo con su criterio y que es evaluada en una escala de observación que recoge 24 elementos correspondientes a la exploración abdominal $(n=12)$, respiratoria $(n=6)$ y cardiaca $(n=6)$ (Tabla I).
Luego, en presencia del profesor, todo el grupo visiona la película. Una copia se entrega a cada estudiante para que la visione tantas veces como desee.

Se les informa que dos días más tarde tendrá lugar una segunda evaluación en la que deberán hacer una exploración física de acuerdo con los conceptos incluidos en la película.

La tercera evaluación se da al final del período de estancia en el Servicio de Medicina Interna.

\section{Resultados}

Diecinueve estudiantes de medicina de tercer año fueron evaluados sobre sus habilidades en la exploración física en su primer día de clase. En sólo dos pruebas, más del $50 \%$ de los estudiantes tenían las habilidades correctas: solicitar al paciente que respirara profundamente y explorar cuatro posiciones diferentes del tórax posterior con un fonendoscopio. Todas las otras habilidades de exploración objeto de estudio no alcanzaron un promedio del $50 \%$ de correcta realización.

Al cabo de 48 horas, 14 estudiantes realizaron una segunda evaluación, dos días después de su llegada al hospital y de haber recibido una copia de la filmación. Los estudiantes mejoraron en esta segunda evaluación en las diferentes pruebas de exploración física, en una media del 43,4\%.

La evaluación final, tras el período de seis semanas en el hospital, se realizó a 13 estudiantes. Mejoraron en un $14,3 \%$ en comparación con la segunda prueba del tercer día de curso (Tabla II).

\section{Discusión}

\section{Enseñanza de la semiología médica}

En la Facultad de Medicina de la Universitat de Barcelona, el estudiante llega a los hospitales en el tercer año de sus estudios, con muy escasa experiencia clínica. Sería interesante que el contacto con los pacientes y centros de salud se diera de forma habitual desde el inicio de sus estudios de medicina [4].

La formación en semiología médica tiene lugar en el Servicio de Medicina Interna a lo largo de seis semanas. En este tiempo, los estudiantes han de aprender a realizar una historia clínica, la exploración física general y neurológica, interpretación de los análisis de laboratorio y diagnóstico por la imagen y electrocardiográfico.

Diecinueve alumnos del curso de tercer año fueron evaluados en el primer día de su estancia. Si sus 
habilidades en la exploración física ya hubieran alcanzado un buen nivel, no habría sido necesario continuar con las evaluaciones. Sin embargo, sus habilidades en la exploración física eran bajas. En tan sólo dos de los 24 objetivos docentes, el 50\% de los estudiantes tenían conocimientos correctos: pedir al paciente que respire profundamente y después auscultarlo con el fonendoscopio.

Las habilidades en la exploración física se persiguen durante todo el período de seis semanas en el área de hospitalización. Introducir una película corta al inicio de su estancia mejora sus conocimientos en exploración física rápidamente y da mayor confianza para la realización de exploraciones reales con más eficacia y menos estrés.

Las filmaciones docentes se han utilizado en docencia médica para la educación en comunicación, pero también son útiles para el desarrollo de habilidades en exploración física [4].

\section{Estudiante-paciente simulado}

En nuestro grupo docente es habitual utilizar un estudiante para simular un paciente [5]. Aporta ventajas en comparación con la enseñanza con pacientes reales [6]. Los pacientes reales se cansan si la exploración se repite de forma igual varias veces en un corto período. La exploración del estudiante es más económica y menos compleja que con un actor simulado, en especial si la evaluación la hace un observador distinto del paciente simulado [7-9].

\section{Lista de control y elementos de comunicación}

Para la valoración de esta herramienta educativa se ha trazado una lista de procedimientos de exploración. La lista incluye 24 elementos diferentes en tres distintos apartados: exploración abdominal, respiratoria y cardiaca en la secuencia habitual de inspección (palpación, percusión y auscultación). Los elementos de comunicación también están incluidos, ya que la exploración física siempre se realiza en una persona y las habilidades de comunicación son obligatorias.

En nuestro recurso docente, los elementos de comunicación han alcanzado peores resultados que en los elementos no comunicativos. Es posible que los estudiantes tengan dificultades en la explicación de las exploraciones a otros estudiantes. En una nueva edición de la filmación sería necesario dar más énfasis a estos aspectos comunicativos.

Con este estudio se puede concluir que el estudiante de medicina de tercer año llega a la etapa de la semiología de la educación con escasa formación
Tabla I. Elementos de la exploración física objeto de estudio.

Observar con detenimiento la caja torácica posterior

Poner ambas manos en el tórax posterior y pedir al paciente que respire profundamente

Pedir al paciente que diga alguna palabra con ' $r$ ' y recoger las vibraciones vocales

Percutir la caja torácica posterior en al menos

Exploración cuatro posiciones diferentes, dos en cada hemitórax

respiratoria

Pedir al paciente que respire profundamente

... y poner el fonendo en al menos cuatro posiciones diferentes en el tórax posterior

Explicar al paciente el motivo de las exploraciones (al menos dos veces)

Anticipar al paciente las maniobras que se van a realizar (al menos dos veces)

Observar con detenimiento la caja torácica anterior

Palpar el hemitórax izquierdo en busca del lugar del latido cardíaco

Auscultar el corazón en al menos cuatro posiciones:

Exploración focos mitral, aórtico, pulmonar y tricuspídeo

cardíaca

Tomar la tensión arterial

Explicar al paciente el motivo de las exploraciones (al menos dos veces)

Anticipar al paciente las maniobras que se van a realizar (al menos dos veces)

Observar con detenimiento el abdomen

Palpación superficial de forma diferenciada de los nueve espacios del abdomen

Palpación profunda de forma diferenciada de los nueve espacios del abdomen

Palpación selectiva del punto del apéndice en la fosa ilíaca derecha

Palpación selectiva del punto de la vesícula en el hipocondrio derecho

Exploración abdominal

Percutir el hipocondrio izquierdo (cámara gástrica)

Percutir el hipocondrio derecho (hepatomegalia)

Auscultación del abdomen, con presión profunda con el fonendo

Explicar al paciente el motivo de las exploraciones (al menos dos veces)

Anticipar al paciente las maniobras que se van a realizar (al menos dos veces)

estándar en relación con el tema. Una breve filmación es un buen elemento para la formación en la exploración física normal, más rápido que otros sistemas de enseñanza, y genera habilidades permanentes, ya que es posible observar buenos resultados al final del proceso de aprendizaje. 
Tabla II. Resultados de las tres evaluaciones realizadas.




Es importante desarrollar otros elementos educativos para lograr dichas habilidades en pocos días con el fin de aprovechar al máximo el curso.

\section{Bibliografía}

1. Palés-Argullós J, Vallés Segalés A, Cardellach F, GomarSancho C, Estrach MT, Cots JM, et al. Habilidades y procedimientos clínicos básicos a adquirir por los estudiantes de la Facultad de Medicina de la Universidad de Barcelona. Educ Med 2001; 4: 72-81.

2. Martens MJ, Duvivier RJ, Van Dalen J, Verwijnen GM, Scherpbier AJ, Van der Vleuten CP. Student views on the effective teaching of physical examination skills: a qualitative study. Med Educ 2009; 43: 184-91.

3. Delás J, Morcillo C, Martín G, Arboix A, Gil de Bernabé E, Parra O. Elementos diagnósticos en medicina interna 20032010. Educ Med 2012; 15: 167-70.
4. Grant A, Robling M. Introducing undergraduate medica teaching into general practice: an action research study. Med Teach 2006; 28: e192-7.

5. Kronfly E, Delás J, Juncosa S, Blay-Pueyo C, Gràcia LL, Martínez-Carretero JM. El proyecto 'COMCOR'. Análisis de un método basado en pacientes simulados, para la evaluación de estudiantes de tercer curso de medicina. Educ Med 2001; 4: 187-93.

6. Gomar-Sancho C, Palés-Argullós J. ¿Por qué la simulación en la docencia de las ciencias de salud sigue estando infrautilizada? Educ Med 2011; 14: 101-3.

7. Karnath B, Thornton W, Frye AW Teaching and testing physical examination skills without the use of patients. Acad Med 2002; 77: 753.

9. Losh DP, Mauksch LB, Arnold RW, Maresca TM, Storck MG, Maestas RR, et al. Teaching inpatient communication skills to medical students: an innovative strategy. Acad Med 2005; 80: 118-24.

10. Karnath B, Frye AW, Holden MD. Incorporating simulators in a standardized patient exam. Acad Med 2002; 77: 754-5. 